\title{
Birefringence controlled room-temperature picosecond spin dynamics close to the threshold of vertical-cavity surface-emitting laser devices
}

\author{
M. Y. Li, ${ }^{1, a)}$ H. Jähme, ${ }^{1}$ H. Soldat, ${ }^{1}$ N. C. Gerhardt, ${ }^{1}$ M. R. Hofmann, ${ }^{1}$ and T. Ackemann ${ }^{2}$ \\ ${ }^{1}$ Photonics and Terahertz Technology, Ruhr-University Bochum, Bochum D-44780, Germany \\ ${ }^{2}$ SUPA and Department of Physics, University of Strathclyde, Glasgow G4 ONG, Scotland, United Kingdom
}

(Received 16 August 2010; accepted 30 September 2010; published online 12 November 2010)

\begin{abstract}
We analyze the spin-induced circular polarization dynamics at the threshold of vertical-cavity surface-emitting lasers at room-temperature using a hybrid excitation combining electrically pumping without spin preference and spin-polarized optical injection. After a short pulse of spin-polarized excitation, fast oscillations of the circular polarization degree (CPD) are observed within the relaxation oscillations. A theoretical investigation of this behavior on the basis of a rate equation model shows that these fast oscillations of CPD could be suppressed by means of a reduction of the birefringence of the laser cavity. () 2010 American Institute of Physics. [doi:10.1063/1.3515855]
\end{abstract}

The study of spin-controlled optoelectronic devices has been a field of very active research over the last decade. Most studies concentrate on light emitting diodes (LEDs) with spin injection. ${ }^{1-5}$ Though polarization degrees up to $32 \%$ have been reported at room-temperature for spin-LEDs, ${ }^{6}$ such values are too low for practical applications in communication technology. In addition, the LED dynamics is generally too slow to allow modulation at the high speed needed for data communication. In contrast, spincontrolled vertical-cavity surface-emitting lasers (VCSELs) can potentially be modulated at high speed and incorporate an intrinsic mechanism for the amplification of spin information due to their nonlinearity at threshold. ${ }^{7,8}$ The implementation of first electrically pumped spin-VCSELs, ${ }^{9,10}$ though only operated at low temperatures, demonstrates that spinVCSELs have enormous potential to become realistic spinoptoelectronic devices. However, conventional electrically pumped VCSELs are known to show complex polarization dynamics, including a predetermination for linearly polarized emission and irregular polarization switching behavior due to cavity anisotropies such as birefringence and dichroism. ${ }^{11-15}$ These cavity anisotropies can possibly suppress the desired spin control effects, making spin-VCSELs potentially useless. In a recent letter, we studied the time-integrated polarization degree of commercial electrically pumped VCSELs and showed that even dominantly electrically pumped devices can show spin dynamics with additional spin-polarized optical excitation. ${ }^{16}$ In this letter we present a time-resolved analysis of the complex polarization dynamics of spinpolarized VCSELs close to threshold and discuss ultrafast modulation scenarios in future spin-VCSELs. ${ }^{17,18}$

Since no electrically pumped room-temperature spinVCSEL device is available yet, we chose a hybrid excitation scheme. The device under study is a commercial electrically pumped GaAs-QW VCSEL (Emcore, Albuquerque, NM, USA, type 8085-2010), Albuquerque, NM, USA, with an emission wavelength of $830 \mathrm{~nm}$ at room-temperature. In addition to standard electrical pumping we use circularly polarized (circ. pol.) light pulses from a Ti:sapphire laser to inject spin-polarized carriers into the active region. The mode-

${ }^{a)}$ Electronic mail: mingyuan.li@rub.de. locked Ti:sapphire laser emits laser pulses at $770 \mathrm{~nm}$ with a pulse width of $80 \mathrm{fs}$ and a repetition rate of $75.5 \mathrm{MHz}$. After propagating through a single mode fiber, installed for reasons of flexibility and beam shaping, ${ }^{16}$ the laser pulses are broadened to approximately 3 ps. The polarization state of the excitation can be controlled by using a pair of retardation plates. The Ti:sapphire optical excitation is incident on the VCSEL surface at a angle of less than $0.4^{\circ}$ to the normal, small enough such that the optical selection rules for excitation normal to the plane of a GaAs quantum well are still valid. $^{19}$ The VCSEL emission is analyzed polarization- and time-resolved by a polarization analyzer based on a Stokes polarimeter followed by a streak camera system with 2 ps time resolution. A schematic of the corresponding experimental setup is shown in Fig. 1 and further details can be found in Ref. 16. All experiments were performed at roomtemperature and without any external magnetic fields.

While the electrical excitation introduces a statistical mixture of spin-up and spin-down electrons with an average spin polarization of zero in the active region of the device, the circularly polarized optical excitation introduces spinpolarized electrons due to the optical selection rules. ${ }^{19}$ To observe spin-induced dynamics of the VCSEL emission, we operate the VCSEL with electrical excitation in the vicinity of the electrical threshold (95\%-105\% of the threshold

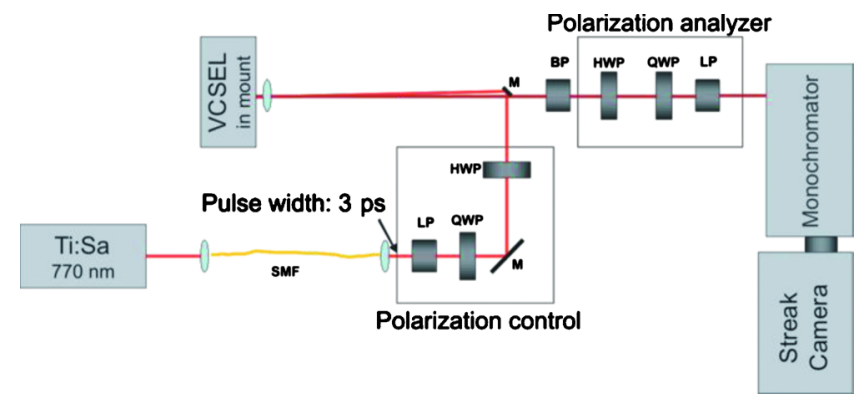

FIG. 1. (Color online) The experimental setup for the time-resolved polarization measurements: (M) mirror, (BP) bandpass, (HWP) half wave plate, (QWP) quarter wave plate, (LP) linear polarizer, and (SMF) single mode fiber. 


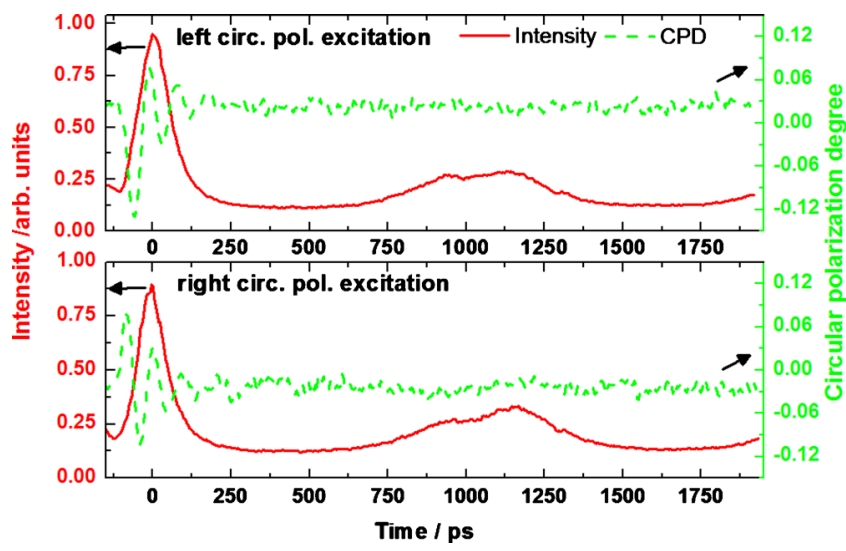

FIG. 2. (Color online) CPD of VCSEL emission upon left (top) and right (bottom) circularly polarized optical excitation. The total intensity relaxation (red solid) is measured by the streak camera. The CPD (green dashed) is calculated from the intensities of left and right circularly polarized emission.

current without optical excitation $)^{20}$ and about $150 \mathrm{~mW}$ (photon density of $6.8 \times 10^{15} / \mathrm{cm}^{2}$ per pulse) of additional circularly polarized optical excitation. Figure 2 shows the dynamics of the total intensity (solid lines) and the calculated time-resolved circular polarization degree (dashed lines) under left (top) and right (bottom) circularly polarized excitation. The intensity dynamics exhibits a first pulse of $150 \mathrm{ps}$ width. After about 900 ps, a weak second pulse appears and indicates the onset of relaxation oscillations. The nonzero background is due to spontaneous emission below lasing threshold. The dynamics of the circular polarization degree (CPD) differs considerably from the intensity dynamics: It exhibits a fast oscillation on a time scale shorter than 100 ps which corresponds to an oscillation frequency higher than 10 $\mathrm{GHz}$ within the first intensity peak. The peak CPD values reach about $10 \%$. The first peak of the CPD is determined by the handedness of the excitation polarization: with a left circularly polarized optical excitation the first peak of the CPD is negative, i.e., the output emission is also left circularly polarized. After approximately 50 ps the CPD flips to a positive peak, the output emission is right circularly polarized. In contrast, the first peak of the CPD with right circularly polarized optical excitation is positive and changes its sign 50 ps later. In both cases, the dynamics of the output CPD lasts only during the first intensity pulse. This time-resolved analysis shows that our experimental procedure controls the polarization of the VCSEL output by the spin-polarized excitation as concluded earlier from time-integrated measurements. ${ }^{16}$ The frequency of oscillations does not depend on excitation conditions within the experimental accuracy. Accordingly, emission of only one circular polarization as it may be desired for applications cannot be easily achieved with the control parameters available in the present setup (see below).

To understand the underlying mechanisms for this behavior we perform numerical calculations based on a spin flip model developed by San Miguel et al. ${ }^{13,21}$ It describes the dynamics of the complex amplitudes $E_{ \pm}$of both circularly polarized optical modes with the corresponding intensities $I_{ \pm}=\left|E_{ \pm}\right|^{2}$, of the normalized carrier density $N$, and of the polarization imbalance $m_{z}$ between the two spin subbands in a coupled rate equation system,

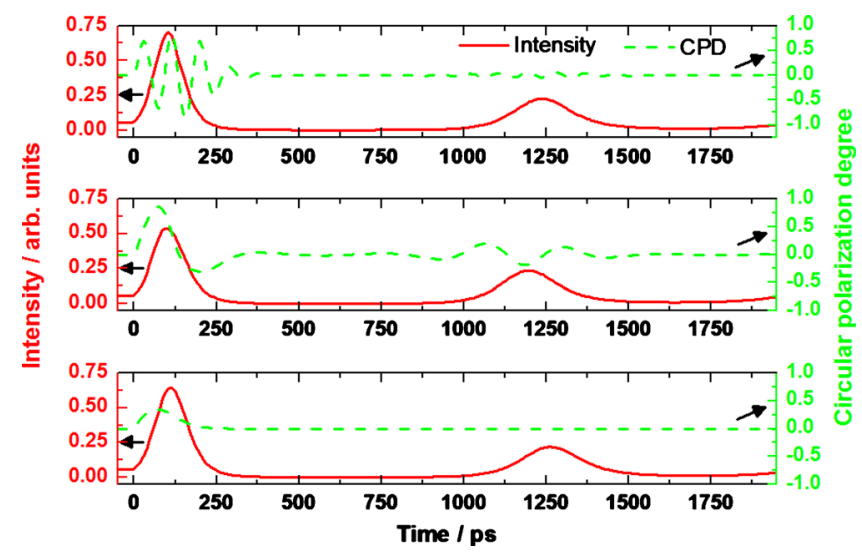

FIG. 3. (Color online) Both calculated intensity (red solid) and CPD (green dashed) dynamics as time dependent functions with different value of birefringence (top $\gamma_{p}=11 \pi \mathrm{GHz}$, middle $\gamma_{p}=3 \pi \mathrm{GHz}$, bottom $\gamma_{p}$ $=0.1 \pi \mathrm{GHz}$ ).

$$
\begin{aligned}
\dot{E}_{ \pm}= & \kappa(1+i \cdot \alpha)\left(N \pm m_{z}-1\right) E_{ \pm}-\left(\gamma_{a}+i \cdot \gamma_{p}\right) E_{\mp} \\
& +\xi_{ \pm} \sqrt{\beta \cdot \gamma \cdot\left(N \pm m_{z}\right)}, \\
\dot{N}= & \gamma\left[\eta_{+}+\eta_{-}\left(1+I_{+}+I_{-}\right) N-\left(I_{+}-I_{-}\right) m_{z}\right], \\
\dot{m}_{z}= & \gamma\left(\eta_{+}-\eta_{-}\right)-\left[\gamma_{s}+\gamma\left(I_{+}+I_{-}\right)\right] m_{z}-\gamma\left(I_{+}-I_{-}\right) N .
\end{aligned}
$$

$\eta_{ \pm}$are pump terms for the spin up (right circ. pol.) and down (left circ. pol.) carrier reservoirs consisting of a background cw term describing the electrical excitation and a time dependent part describing the optical excitation, $\alpha$ is the linewidth enhancement factor, $\beta$ is the spontaneous emission factor, $\xi_{ \pm}$is the noise source, $\kappa$ is the cavity decay rate, $\gamma$ is the carrier density decay rate, $m_{z}$ is the carrier spin magnetization with the decay rate $\gamma_{s}$ due to the spin flip processes, $\gamma_{p}$ is the linear birefringence, and $\gamma_{a}$ is the linear dichroism. This model allows the analysis of the interplay between CPD dynamics, carrier densities, and spin-polarized excitation under the conditions of our experiment. The following parameters for the calculations are taken from earlier work on similar VCSEL devices ${ }^{11}$ and were adapted to the present model:

$$
\begin{aligned}
& \gamma=1.5 \mathrm{~ns}^{-1}, \quad \gamma_{s}=40 \mathrm{~ns}^{-1}, \quad \gamma_{p}=11 \pi \mathrm{GHz}, \\
& \gamma_{a}=1.6 \pi \mathrm{GHz}, \quad \kappa=300 \mathrm{~ns}^{-1}, \quad \alpha=2, \quad \beta=10^{-6} .
\end{aligned}
$$

Figure 3 shows the results of our calculations. In all cases, the spin unpolarized excitation is $105 \%$ of the threshold and the spin-polarized excitation [a Gaussian pulse with a pulse duration of 3 ps, a peak power of 13.3 times the threshold and a CPD of +0.5 (right circ. pol.), which is the typical value of optical pumping in GaAs-QWs] is then injected at 0 ps. In Fig. 3 (top), we use the parameters given above. The experimentally observed dynamics of output intensity and CPD are very well reproduced. Just as in the experiment a strong CPD oscillation is found within the first intensity pulse. This CPD oscillation reaches a peak-value of 0.8 , damps out in the tail of the first intensity pulse, and is hence not present in the second intensity pulse. The observed dynamics is a consequence of the interplay of the spin dynamics with birefringence in the laser cavity, which couples the circular polarization components of different helicity and hence leads to an oscillation between them. This transition occurs because the spin populations are depleted in an aniso- 
tropic way and this anisotropy feeds back into the dynamics due to birefringence. These effects were observed before only indirectly in the noise spectrum of linearly polarized states $^{14,18,22}$ and in the transient of a polarization switching between linearly polarized states. ${ }^{15}$ Here we demonstrate them directly as large amplitude oscillations between circularly polarized states.

The switching rate is usually given by the linear birefringence and small corrections due to nonlinear spin effects. ${ }^{23,24}$ Both our experimental and theoretical observations show that the CPD oscillation frequency has a negligible dependence on the exact pumping conditions, i.e., the contributions due to nonlinear spin effects are very small in our case. The direct connection between linear birefringence and oscillation frequency provides a possibility to reduce the oscillation, in order to get purely circularly polarized emission by tuning the birefringence. Reducing the birefringence in the device should potentially result in pure spin oscillation free emission of the spin-polarized VCSEL. To prove this, we have varied the birefringence in the numerical model. In Fig. 3 (middle and bottom) we show the intensity and CPD dynamics with the same parameters as in Fig. 3 (top) except for the birefringence being reduced to $\gamma_{p}=3 \pi \mathrm{GHz}$ (middle) and $\gamma_{p}=0.1 \pi \mathrm{GHz}$ (bottom). Obviously, with lower $\gamma_{p}$ the frequency of the CPD oscillation is lower and less peaks of CPD occur inside the first intensity pulse. With $\gamma_{p}$ $=0.1 \pi \mathrm{GHz}$, the first intensity pulse is only right circularly polarized, as intended. The CPD peak-value above 0.3 could be increased with a spin-polarized excitation that is higher relative to the unpolarized electrical injection. The calculations with slightly varying either spin-polarized (peak power from 10 to 20 times the threshold) or unpolarized (cw power from $98 \%$ to $107 \%$ of the threshold) excitation show similar scenarios: the CPD dynamics appears only inside the first intensity pulse and is strongly suppressed inside the following pulse. The frequency of the CPD oscillations does not change significantly upon these variations of the pumping conditions.

In conclusion, we have analyzed the polarization dynamics of electrically pumped VCSELs with additional optically induced spin injection. The dynamics was investigated experimentally and theoretically on a picosecond time scale. The circular polarization dynamics can be significantly faster than the intensity dynamics depending on the birefringence in the cavity. We observed fast oscillations of the circular polarization degree at a frequency higher than $10 \mathrm{GHz}$. Theoretical simulations demonstrate that the experimentally observed dynamics is a consequence of the interplay of the spin dynamics with birefringence, which couples the circular polarization components of different helicity and hence leads to a switching between them. By tuning the birefringence, e.g., by applying additional strain to the laser device, ${ }^{24}$ the oscillations can be reduced to a single circularly polarized pulse emission, controlled by the spin orientation of a small amount of injected carrier spins. For future electronically pumped spin-VCSELS ${ }^{9}$ our findings imply that the birefringence in the devices has to be even more carefully controlled than in conventional VCSELS in order to fully benefit from the high potential of spin-induced dynamics.

The authors thank the Deutsche Forschungsgemeinschaft (DFG) for support within the Sonderforschungsbereich 491.

${ }^{1}$ R. Fiederling, M. Keim, G. Reuscher, W. Ossau, G. Schmidt, A. Waag, and L. W. Molenkamp, Nature (London) 402, 787 (1999).

${ }^{2}$ Y. Ohno, D. Y. Young, F. Matsukura, H. Ohno, and D. D. Awschalom, Nature (London) 402, 790 (1999).

${ }^{3}$ H. J. Zhu, M. Ramsteiner, H. Kostial, M. Wassermeier, H.-P. Schönherr, and K. H. Ploog, Phys. Rev. Lett. 87, 016601 (2001).

${ }^{4}$ T. Hanbicki, B. T. Jonker, G. Itskos, G. Kioseoglou, and A. Petrou, Appl. Phys. Lett. 80, 1240 (2002).

${ }^{5}$ N. C. Gerhardt, S. Hövel, C. Brenner, M. R. Hofmann, F.-Y. Lo, D. Reuter, A. D. Wieck, E. Schuster W. Keune, and K. Westerholt, Appl. Phys. Lett. 87, 032502 (2005).

${ }^{6}$ X. Jiang, R. Wang, R. M. Shelby, R. M. MacFarlane, S. R. Bank, J. S. Harris, and S. S. P. Parkin, Phys. Rev. Lett. 94, 056601 (2005).

${ }^{7}$ S. Hövel, N. Gerhardt, M. Hofmann, J. Yang, D. Reuter, and A. Wieck, Electron. Lett. 41, 251 (2005).

${ }^{8}$ N. Gerhardt, S. Hövel, M. Hofmann, J. Yang, D. Reuter, and A. Wieck, Electron. Lett. 42, 88 (2006).

${ }^{9}$ M. Holub, J. Shin, D. Saha, and P. Bhattacharya, Phys. Rev. Lett. 98 146603 (2007).

${ }^{10}$ D. Basu, D. Saha, C. C. Wu, M. Holub, Z. Mi, and P. Bhattacharya, Appl. Phys. Lett. 92, 091119 (2008).

${ }^{11}$ M. Sondermann, M. Weinkath, and T. Ackemann, IEEE J. Quantum Electron. 40, 97 (2004).

${ }^{12}$ T. Ackemann and M. Sondermann, Appl. Phys. Lett. 78, 3574 (2001).

${ }^{13}$ M. San Miguel, Q. Feng, and J. Monoley, Phys. Rev. A 52, 1728 (1995).

${ }^{14}$ M. van Exter, M. Willemsen, and J. P. Woerdman, Phys. Rev. A 58, 4191 (1998).

${ }^{15}$ M. Willemsen, M. van Exter, and J. P. Woerdman, Phys. Rev. Lett. 84 4337 (2000)

${ }^{16}$ S. Hövel, N. C. Gerhardt, M. R. Hofmann, F.-Y. Lo, D. Reuter, A. D Wieck, E. Schuster, H. Wende, W. Keune, O. Petracic, and K. Westerholt, Appl. Phys. Lett. 92, 242102 (2008).

${ }^{17}$ S. Hallstein, J. D. Berger, M. Hilpert, H. C. Schneider, W. W. Rühle, F. Jahnke, S. W. Koch, H. M. Gibbs, G. Khitrova, and M. Oestreich, Phys. Rev. B 56, R7076 (1997)

${ }^{18}$ E. Blansett, M. Raymer, G. Khitrova, H. Gibbs, D. K. Serkland, A. Allerman, and K. Geib, Opt. Express 9, 312 (2001).

${ }^{19}$ M. Dyakonov and V. Perel, Optical Orientation (Elsevier Science, Netherlands, 1984).

${ }^{20}$ The threshold current of the VCSEL decreasing with increasing temperature, due to the sign of the detuning between the gain maximum and the cavity mode. This leads to an improved overlap of cavity mode and gain maximum above room-temperature. Upon optical excitation even in pulsed operation, heating of the device cannot be completely avoided Therefore, the optical excitation does not only provide additional spinpolarized carriers but also slightly reduces the threshold for electrical excitation.

${ }^{21}$ A. Gahl, M. San Miguel, and S. Balle, IEEE J. Quantum Electron. 35, 342 (1999).

${ }^{22}$ H. Hofmann and O. Hess, Phys. Rev. A 56, 868 (1997).

${ }^{23}$ M. Van Exter, M. A. Jansen van Doorn, and J. P. Woerdman, Phys. Rev. A 56, 845 (1997).

${ }^{24}$ A. K. Jansen van Doorn, M. P. van Exter, and J. P. Woerdman, Appl. Phys. Lett. 69, 1041 (1996). 